\title{
Einkommens- und Vermögensungleichheit in einem wohlhabenden Staat
}

Einkommens- und Vermögensungleichheit wurde von vielen Ökonomen lange als soziales oder politisches Problem behandelt, das die Ökonomie wenig angehe. In letzter Zeit wird jedoch auch in den Wirtschaftswissenschaften darüber diskutiert, dass zu große Ungleichheit zum Wachstumshemmnis werden kann. Doch schon bei der Beschreibung des Phänomens beginnen die Wirtschaftswissenschaftler zu streiten, etwa ob die Ungleichheit wirklich wächst und welche Rolle die wahrgenommene Ungleichheit spielt. Die lange als sicher geltende Formel, dass mehr Gleichheit nur durch weniger Effizienz "erkauft" werden könne, entspricht nicht mehr dem Stand der Forschung, und es sind dringend neue Sichtweisen, Ursachenanalysen und wirtschaftspolitische Vorschläge gefragt.

Eigentlich wären bei wachsendem Wohlstand in Deutschland Verteilungsspielräume vorhanden, die eine angemessene Teilhabe aller an steigenden Einkommen und Vermögen erlauben sollten. Doch offensichtlich passiert das nicht. Das Bildungssystem scheint soziale Strukturen eher zu reproduzieren, statt innen zu mehr Durchlässigkeit zu verhelfen. Packt die Politik in ihren verteilungspolitischen Konzepten alles richtig an? Reicht es, die Einkommen, so wie sie aus Marktprozessen entstehen, umzuverteilen, oder ist bereits in der Entlohnung eine Schieflage zu entdecken? Wie gut gelingt die Integration von Arbeitslosen und Frauen in den Arbeitsmarkt? Warum fällt Deutschland in der Vermögensbildung so deutlich hinter vergleichbare Länder zurück? Zu diesen und weiteren Fragen lieferte die Konferenz, die der Wirtschaftsdienst im November 2015 zusammen mit dem Deutschen Institut für Wirtschaftsforschung in Berlin veranstaltete, interessante Beiträge. Die Vorträge der Konferenz werden in diesem Heft als wissenschaftliche Aufsätze dokumentiert.

Dass politischer Handlungsbedarf besteht, wird aus den Ausführungen von Marcel Fratzscher deutlich. Nicht nur bei den Einkommen sieht er Probleme für Wirtschaft und Gesellschaft, sondern auch bei den in Deutschland extrem ungleich verteilten Vermögen. Hinzu kommt eine geringe soziale Mobilität. Beides hängt zusammen: Bei niedrigen Einkommen und wenig Vermögen können Familien weder in die Ausbildung ihrer Kinder ausreichend investieren noch im erforderlichen Umfang für das Alter vorsorgen. Es entstehen Nachteile für Wirtschaft und Gesellschaft durch nicht erschlossene Qualifikationspotenziale (d.h. hinter den Möglichkeiten zurückbleibende Produktivitätsentwicklung) und durch Altersarmut, zu deren Ausgleich der Staat erhebliche Mittel aufwenden muss, die an anderer Stelle für Investitionen fehlen. Insofern käme eine effektive Umverteilungspolitik sowohl den Beziehern geringer Einkommen als auch der Wirtschaft zugute. Hier sieht Fratzscher noch deutliche Defizite in der deutschen Wirtschaftspolitik.

Der Blick des Politikers auf die Einkommensunterschiede in Deutschland ist naturgemäß ein anderer. Jens Spahn, Parlamentarischer Staatssekretär im Bundesfinanzministerium, weist auf die Verbesserung der Situation bei den verfügbaren Einkommen in den letzten Jahren hin und betont die Erfolge der ausgewogenen Verteilungspolitik. Hier gelte es insbesondere, die Umverteilung so zu steuern, dass Leistungsanreize bestehen bleiben, die bei einer zu egalitären Verteilung wegfallen könnten. Die Politik setze daher darauf, mehr Menschen in Arbeit zu bringen und die Vermögensbildung zu fördern.

Wichtig für politische Entscheidungen ist, wie die Verteilung von Einkommen und Vermögen in der Bevölkerung empfunden wird. Judith Niehues' Beitrag beschäftigt sich mit dieser Frage. Auf der Basis einer internationalen Studie zeigt sie, dass die subjektive Einschätzung der Verteilungssituation höchst selten mit der tatsächlichen übereinstimmt. In Ländern mit relativ gleicher Verteilung wird die Ungleichheit oft größer eingeschätzt, als sie tatsächlich ist. Umgekehrt empfinden die Bürger in Ländern mit großen Einkommensunterschieden die Verteilung als ausgewogener. Dies liegt zum einen daran, dass die subjektive Einschätzung der Ungleichheit von der absoluten Höhe der Einkommen und Vermögen abhängt, und zum anderen, ob die eigene Gesellschaft als "gerecht" oder "ungerecht" beurteilt wird. Generell gilt, dass die Ungleichheit subjektiv als gravierender empfunden wird, als sie tatsächlich ist. Dies lässt sich jedoch nicht systematisch nachweisen.

Herrscht Unklarheit darüber, wie die eigene Gesellschaft dasteht, hilft oft ein Blick über die Grenzen. Den liefert der Beitrag Horacio Levys von der Social Policy Division der OECD. Levy untermauert mit international vergleichbaren Daten viele Erkenntnisse anderer Wissenschaftler. So kann etwa gezeigt werden, dass die einfache Vorstellung, mehr Gleichheit gehe mit geringerer Effizienz einher, relativiert werden muss, oder dass der Trend zunehmender Ungleichheit, der in Vorkrisenzeiten schon galt, sich in der Krise deutlich fortsetzt. Betroffen sind besonders die untersten 
Einkommensschichten, aber auch zunehmend die untere Mittelschicht, die langfristig wenig vom Wachstum profitiert. Das Fazit der OECD lautet, dass mehr Gleichheit allen nützt. Eine hohe Qualität von Arbeitsplätzen (hohes durchschnittliches Qualifizierungsniveau, angemessene Bezahlung) wirkt sich ebenso positiv aus wie eine höhere Erwerbsbeteiligung von Frauen. Vermehrte Anstrengungen bei Bildung und Erziehung befördern eine angemessene Verteilung und gleichzeitig Wachstum, und eine Reform der Steuer- und Sozialprogramme kann die Effizienz der Umverteilung erhöhen.

Der Aufsatz von Gerhard Bosch „Einkommensentstehung als Verteilungsfaktor" fokussiert auf die Verteilung der Primär- oder Markteinkommen. Betrachtet man lediglich die Einkommensverteilung nach der Umverteilung, wird das Ausmaß der Ungleichheit unterschätzt, da viele Haushalte aus den unteren Einkommensschichten nur durch ergänzende Zahlungen des Staates in höhere Schichten wechseln. Eine ausgewogenere Verteilung der Primäreinkommen entlastet den Staat, der dadurch weniger Ressourcen zur Vermeidung von steigender Armut aufwenden muss. Zudem ist eine Lösung des Verteilungsproblems über eine Angleichung der Primäreinkommen statt über sozialpolitische Maßnahmen integrationsfördernd, da Bürger, die ihren Lebensunterhalt selbst verdienen, sich intensiver am gesellschaftlichen Leben beteiligen. Die Ungleichheit der Primäreinkommen führt zu einer Verzerrung des Wettbewerbs zwischen Unternehmen, da diejenigen, die Löhne unterhalb des Existenzminimums zahlen, sich über die Subvention der Löhne durch staatliche Transfers Vorteile verschaffen. Bemerkenswert ist, dass der Trend zu einem Auseinanderdriften der Primäreinkommen bereits seit über 20 Jahren anhält und sich relativ unabhängig von Änderungen der Gesetzgebung oder Wachstumsverläufen fortsetzt. Ergebnis ist eine deutlich sichtbare Schrumpfung der mittleren Einkommensgruppen. Trotz der großen Bedeutung von Umverteilungsmaßnahmen für die Stabilität der Gesellschaft kommt daher einer Politik, die auf eine gleichmäßigere Verteilung der Primäreinkommen zielt, große Bedeutung zu. Oft werden sich ändernde Qualifikationsanforderungen, Strukturentwicklungen und Globalisierung als Erklärung für wachsende Ungleichheit angeführt. Demgegenüber verweist Bosch auf die Bedeutung der Machtposition der Arbeitgeber bei der Lohnsetzung.

Norbert Berthold betrachtet zunächst die Unterschiede in der Entwicklung von Markt- und Nettoeinkommen. Da die Verteilung der Nettoeinkommen sich langfristig kaum verändert hat, bei den Markteinkommen aber eine deutliche Spreizung stattgefunden hat, muss in Deutschland die Intensität der Umverteilung zugenommen haben. Berthold untersucht folgerichtig die politischen Determinanten von Umverteilung. Angelehnt an Meltzer und Richard wird das Umverteilungsvolumen vom Wählerverhalten und der Reaktion der Politik
Dr. Brigitte Preissl ist Chefredakteurin der Zeitschriften Wirtschaftsdienst und Intereconomics und leitet den Bereich Wissenstransfer Wirtschaftswissenschaften in der ZBW - Leibniz-Informationszentrum Wirtschaft in Hamburg.

darauf abgeleitet. Grundsätzlich gilt, je mehr Demokratie, desto mehr wird umverteilt. Dabei spielt die subjektive Einschätzung der Verteilung durch die Bevölkerung - und hier insbesondere durch die Mittelschicht - eine große Rolle. Denn an ihr orientiert sich die Politik und wird im Hinblick auf die nächsten Wahlen aktiv, um eine durch zu große Ungleichheit hervorgerufene Unzufriedenheit mit der Regierung zu vermeiden. Da die Einschätzung der Betroffenen nicht immer den tatsächlichen Verhältnissen entspricht, ist das Ergebnis suboptimal. Interessant ist der Grad der Abweichung von gefühlter und tatsächlicher Ungleichheit in verschiedenen Ländern. Hier sind die Befunde sehr unterschiedlich.

Ebenso wie Bosch beklagt auch Joachim Möller in seinem Aufsatz „Lohnungleichheit: Gibt es eine Trendwende?" das Festhalten der Ökonomenzunft an dem Glauben, Ungleichheit fördere die Effizienz in einer Volkswirtschaft. In seiner empirischen Analyse zeigt Möller die Entwicklung der Lohnspreizung seit 1990, die von führenden Ökonomen zunächst ausdrücklich begrüßt wurde. Er hebt die Bruttoverdienstlücke zwischen Männern und Frauen und das deutlich stärkere Auseinanderdriften der Löhne bei Frauen in Ostdeutschland hervor. Schließlich wendet er sich den Ursachen für die Entwicklung der Verteilung seit 1995 zu und identifiziert qualifikatorische, institutionelle und auf die Bewertung von Arbeitsleistung zurückgehende Effekte. Dass die Ungleichheit seit 2010 weniger schnell wächst, könnte auch auf das Mindestlohngesetz sowie auf die gezielte Förderung von Frauen zurückzuführen sein.

Mit den in diesem Heft publizierten Aufsätzen werden wesentliche Aspekte der Verteilung von Einkommen und Vermögen thematisiert, empirische Entwicklungen aufgezeigt, Ursachen und Erklärungsmuster analysiert. Dabei gibt es bei einigen zentralen Erkenntnissen einen erstaunlichen Grad an Konsens unter den Autoren. Dazu gehört etwa, dass eine Verringerung der Ungleichheit der Wirtschaftsentwicklung zugutekommt und ihr nicht schadet, dass sowohl die Primäreinkommen als auch die Einkommen nach Verteilung zu betrachten sind, das erste, um die Ursachen von unangemessener Ungleichheit zu erkennen, das zweite, um die Effizienz und Effektivität der Umverteilung zu beurteilen. Daraus leiten sich Handlungsfelder ab, die über den üblichen Kanon der Verteilungspolitik hinausgehen. 